\title{
LOS PROBLEMAS DERIVADOS DE LA COEXISTENCIA DE LAS REGLAMENTACIONES SOBRE LOS CREDITOS DOCUMENTARIOS STAND-BY Y SOBRE LAS GARANTIAS INDEPENDIENTES
}

\author{
Javier San Juan Crucelaegui \\ Profesor de la Universidad de Deusto
}

\begin{abstract}
Sumario: I. La naturaleza y modalidades de los créditos stand-by. 1. El crédito stand-by financiero. 2. La utilización del crédito stand-by en garantía del pago del precio. II. La diferente naturaleza jurídica de los créditos documentarios ordinarios y de los créditos stand-by. 1. Las diferentes finalidades económicas. 2. El diferente grado de formalismo. III. La aplicabilidad de las reglas y usos uniformes sobre los creditos documentarios (RUU) a los créditos stand-by. 1. Los preceptos de las RUU aplicables a los créditos stand-by. 2. Los preceptos de las RUU no aplicables a los créditos stand-by. IV. La identidad de naturaleza entre las garantías independientes y los créditos stand-by y el campo de aplicación de sus respectivas reglamentaciones. 1. La inaplicabilidad de las RUU a las garantías independientes. 2. La aplicabilidad de las Reglas Uniformes sobre las Garantías a Demanda (RUGD) a los créditos stand-by.
\end{abstract}

En las actuales relaciones mercantiles internacionales se ha producido una mutación y una ampliación de los riesgos asumidos por el acreedor internacional y, por ende, ha surgido la necesidad de garantía de tales riesgos transformados e intensificados. Los ordenamientos jurídicos no han podido servir esta nueva necesidad por recurso a los tipos contractuales por ellos conocidos y aceptados, fueren de tradicional raigambre o de reciente aparición y consolidación. El mecanismo del crédito documentario, aun habiendo alcanzado una plena decantación jurídica, no se acomoda a la cobertura de riesgos complejos y perdurables. Por otra parte, y en términos generales, no resultan de interés para el acreedor las figuras tradicionales de garantía, marcadas primordialmente por su carácter de accesoriedad, porque el garante accesorio puede invocar las excepciones y medios de defensa que corresponden al deudor. En ese caso, el acreedor viene obligado con frecuencia a la incoación de procedimientos judiciales que entrañan dilaciones e in- 
convenientes. Por otro lado, las instituciones financieras, usualmente libradoras de las garantías en las relaciones internacionales, se resisten a actuar como garantes accesorios, pues ello implica asumir la dificultad de la determinación de aquellas situaciones en las que deben proceder a la realización del pago, pudiendo verse involucradas en las diferencias que pudieran oponer a las partes en relación con el contrato subyacente garantizado.

Tradicionalmente, el género de las garantías personales ha contenido en su seno la sola especie de la fianza, tipo jurídico decisivamente teñido por su carácter de accesoriedad, y figura contractual que no ha recibido atención primordial en el pasado en orden a su utilización en el comercio internacional. Por el contrario, en los últimos años las necesidades de nuevas figuras internacionales de garantía han convertido a la fianza en centro de atención y ha adquirido rango de garantía de primer orden. Sin embargo, la rigidez de su regulación y la tendencia de la jurisprudencia a la firme y nítida protección de los caracteres de la fianza, hasta llegar a entenderlos como inderogables, han provocado una consecuencia de opuesto signo, arrastrando la crisis de la institución al causar su incapacidad para la satisfacción de aquellas necesidades para las que su utilización era pretendida. Dicha crisis se manifiesta en que la regulación jurídica de la fianza puede producir efectos imprevisibles, lo que entraña mayor gravedad puesto que su finalidad estriba, precisamente, en procurar una garantía. La historia del desarrollo de la fianza pone de manifiesto que el exceso de protección del tipo contractual y de sus caracteres frente a los intereses del acreedor garantizado se traduce inevitablemente en desconfianza hacia una figura de garantía que se ha convertido en aleatoria, pues, en virtud de la comunicación de excepciones entre relación garantizada y relación de garantía, puede acabar por significar garantía de la ejecución de una sentencia judicial. Aun pudiendo incurrir la siguiente afirmación en exageración, se tiene la impresión de que el respeto a la obligación asumida no siempre se produce cuando se recurre a la fianza. Por ello, no debe causar extrañeza que los acreedores insatisfechos y desencantados busquen nuevas fórmulas de garantía.

Descartada la utilización de la figura de la fianza para el aseguramiento de las transacciones internacionales como consecuencia de su carácter accesorio, la aparición de la figura de las garantías independientes constituye, en tal contexto, la réplica de los importadores frente al rigor de las condiciones de pago impuestas por los exportadores, generalmente consistentes en la exigencia del pago de la compraventa mediante crédito documentario. Frente a la seguridad así obtenida por el exportador, el importador desea verse igualmente protegido por una 
promesa de pago independiente del contrato subyacente y emitida por una institución financiera que suscita confianza y que permanece al margen y desconectada de la relación subyacente garantizada.

En respuesta a tal nueva necesidad de figuras de garantía que superen las insuficiencias de las tradicionales, se ha producido la aparición de las hoy llamadas garantías independientes, con frecuencia denominadas «garantías a demanda» o «a primer requerimiento». Las garantías independientes europeas y su equivalente norteamericano, las stand-by letters of credit, constituyen un fenómeno jurídico relativamente nuevo pues, aun cuando parece que su uso se produjo inicialmente en los mercados internos de los EEUU a lo largo de la década de los años sesenta, su utilización generalizada en las relaciones internacionales se produjo en la década de los años setenta ${ }^{1}$. En aquellas fechas, el espectacular incremento de los ingresos financieros recibidos por los países productores de petróleo del Oriente Medio les permitió la conclusión de múltiples y complejos contratos con empresas occidentales para la construcción y mejora de infraestructuras, construcción de equipamientos públicos, puesta en marcha de proyectos industriales y agrícolas e impulso de proyectos de defensa nacional. Probablemente, el origen general de las garantías independientes y, más en particular, el de las garantías pagaderas a demanda simple, puede ser situado en dichas transacciones. Su utilización acabaría por alcanzar, posteriormente, amplísima extensión. Su expansión se explica, asimismo, por el hecho de que pueden ser utilizadas para la cobertura de toda clase de transacciones, fueren éstas de índole financiera o no. En suma, mientras la posición del vendedor o exportador había quedado garantizada desde antes incluso de la década de los años cincuenta mediante la figura del crédito documentario, la posición del comprador o importador no había encontrado auténtico aseguramiento hasta el inicio de la década de los años setenta $^{2}$. Sin embargo, puede afirmarse que, en la actualidad, la mayor parte de las transacciones internacionales se llevan a cabo mediante la inclusión de algún tipo de garantía para el aseguramiento de la posición del adquirente.

En consonancia con tal evolución, la Cámara de Comercio Internacional ha preparado un conjunto de reglamentaciones a propósito de las

1 Acerca del nacimiento de la figura de las garantías abstractas o independientes, vid. Gavalda y Stoufflet, «La lettre de garantie internationale», en Rev. Trimestrielle de Droit Commercial, Tomo XXXIII, 1980, págs. 1-23; asimismo, BERTRAMs, Bank Guaran-

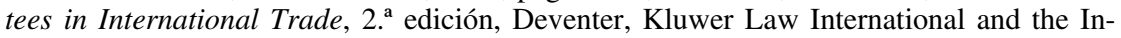
ternational Chamber of Commerce, 1996, págs. 42 y sigs.

2 Acerca del nacimiento del crédito documentario, vid. STOUfFlet, Le crédit documentaire, París, Librairies Techniques, 1957, págs. 42 y sigs. 
garantías contractuales utilizadas en la contratación internacional ${ }^{3}$. En su orden cronológico, el primer texto es el titulado Reglas Uniformes Relativas a las Garantías Contractuales, de 1978, que permanece formalmente vigente a pesar de la falta de aceptación de que fue objeto, lo que fue consecuencia de que dicho texto constituyera una toma de postura por parte de la CCI contra la figura de las garantías abstractas o independientes, pretendiendo con la promulgación del citado texto una «moralización» de la práctica internacional. La realidad de la generalizada aceptación internacional dispensada en favor de las garantías independientes llevó posteriormente a la CCI, en 1992, a la promulgación de las Reglas y Usos Uniformes Relativos a las Garantías a Primer Requerimiento, en las que se dispensa plena acogida a las garantías independientes de acuerdo con la práctica internacionalmente observada. Sin embargo, manteniendo un cierto grado de oposición a las mismas, las configura como primordialmente revestidas de un carácter documentario, asumiendo, en consecuencia, una postura en algún grado contraria a las garantías pagaderas a demanda simple de su beneficiario. Por último, en 1994, bajo las presiones singularmente de algunas empresas constructoras y compañías de seguros, la CCI publicó las reglas sobre los Contract Bonds ${ }^{4}$, lo que contribuyó a la creación de lo que puede parecer una maraña normativa, pero que es reglamentación claramente diferenciada de cualquier otra vigente al regular figuras de garantía caracterizadas por su esencial accesoriedad ${ }^{5}$.

Confusión, asimismo, se ha venido derivando la coexistencia de las Reglas y Usos Uniformes Relativos a los Créditos Documentarios pues, desde la Revisión de 1982, aquellas dieron forzada acogida y extendieron su regulación a los créditos documentarios stand-by, figura ésta que ostenta una clara identidad de naturaleza jurídica con las garantías indepen-

3 Acerca de la labor de la CCI en lo relativo a la unificación del derecho en materia de garantías, vid. SÁNCHEZ CALERO, «Garantías a primer requerimiento», en Las garantías a primer requerimiento (garantías a primera demanda), Fundación BBV-Civitas, Madrid, 1996, págs. 36-38.

4 Traducción al castellano, Publicación CCI, Reglas para las Fianzas Contractuales.

5 Las Naciones Unidas, a través de la Uncitral, dieron fin en 1995 a la Convención en materia de Garantías Independientes, la cual quedó abierta a la firma de los Estados desde el mes de diciembre del citado año. La Convención ha recibido un escaso número de ratificaciones y adhesiones hasta la fecha, por lo que cabe la duda sobre su futuro éxito. Sin embargo, este último texto presenta el especial interés de refundir el concepto y la regulación de las figuras de las garantías independientes y de los créditos documentarios stand-by, aceptando la identidad de naturaleza jurídica de las mismas y superando de esta forma la incertidumbre que pudiera derivarse de las diversas reglamentaciones emanadas de la CCI. 
dientes $^{6}$. Al ser estas últimas objeto de regulación específica en 1992, permaneciendo los créditos documentarios stand-by bajo la disciplina de las RUU, el resultado se traduce en la doble regulación de un mismo mecanismo jurídico de garantía, siendo por ello necesario analizar la coexistencia y delimitar el campo de aplicación de las RUU y de las RUGD.

Las garantías utilizadas en el comercio internacional pueden asumir formas diversas, tales como créditos documentarios comerciales u ordinarios, garantías independientes o cartas de crédito stand-by ${ }^{7}$. Entre las citadas figuras cabe la apreciación de grados de parentesco ${ }^{8}$, puesto que el crédito documentario comercial $\mathrm{u}$ ordinario es el origen de las cartas de crédito stand-by, y éstas parecen serlo, a su vez, de las garantías independientes. La cronología seguida en su utilización parece respaldar la idea de una genealogía de las garantías independientes, según la cual el origen inicial se encontraría en el crédito documentario y los posteriores desarrollos habrían terminado por retornar a sus fuentes originales. Esta idea habría llevado a los redactores de las RUU, al haber apreciado tal parentesco, al sometimiento de ambas figuras a unos mismos principios inspiradores básicos en la Revisión de las Reglas y Usos Uniformes relativas a los créditos documentarios llevada a cabo en 1983, momento en que la figura del crédito documentario stand-by fue recogida en las RUU.

Los créditos stand-by se desarrollaron a partir de los años cincuenta ${ }^{9}$. Han sido utilizados por los bancos norteamericanos tanto en operaciones comerciales internas como en transacciones internacionales, de re-

${ }^{6}$ De otro lado, desde la Revisión de las RUU de 1982, la CCI había dado acogida en las RUU aplicables a los créditos documentarios a la figura de los créditos stand-by, substancialmente similares a las garantías independientes, los cuales disponen así de una regulación diferenciada. Sin embargo, la identidad o similitud conceptuales de ambas figuras plantea la cuestión de la aplicabilidad de las RUU a las garantías independientes en razón de la apuntada similitud conceptual entre ambas.

7 Cfr. Díaz Moreno, «Los trabajos de Uncitral en materia de garantías independientes internacionales», en Rev. de Derecho Mercantil, 1993, págs. 151-205. El citado autor, pág. 152 afirma: «carta de crédito contingente es el término usado como traducción de la expresión inglesa stand-by letters of credit en los documentos de la Uncitral redactados en castellano. Tal traducción no resulta satisfactoria, se resiente de una cierta artificiosidad, y no encuentra precedente o punto de referencia en la terminología generalmente usada, sin que la expresión inglesa haya sido traducida a otros idiomas además de al castellano».

8 Cfr. PuECH, «Credit documentaire, garanties bancaires, stand-by letter of credit: une etude comparative», en Actualites BFCE, julio, 1988, págs. 11-12; SIMLER, Le cautionnement et les garanties autonomes, 2. a edición, París, Litec, 1992, pág. 675.

9 Resulta de especial interés, el informe de la Secretaría General de la Uncitral, XII sesión, Viena, 18 a 29 de junio de 1979, «Lettres de credit standby», Doc. A/CN.)/163, de 9 de mayo de 1979, en Uncitral Yearbook, vol. X, 1979, pág. 86. 
sultas de la prohibición ${ }^{10}$ que sobre ellos pesaba de emitir garantías por cuenta de terceros ${ }^{11}$ lo que resultaba de la exclusiva competencia de las compañías de seguros ${ }^{12}$. Igual prohibición pesaba sobre los bancos de otros países. Ello ha permitido afirmar ${ }^{13}$ que las cartas de crédito standby son el resultado de un camuflaje, consecuencia de la desviación padecida por la técnica del crédito documentario.

Ha venido subsistiendo un grado de confusión en cuanto a la regulación de las diferentes figuras mencionadas y el campo de aplicación de las distintas reglamentaciones emanadas de la $\mathrm{CCI}^{14}$ a tal propósito, pues la CCI dio cuerpo a regulaciones distintas aplicables a las garantías independientes y a los créditos stand-by al decidir la inclusión de éstos en las RUU aplicables a los créditos documentarios ${ }^{15}$, de donde se deducía

10 Tal prohibición lo fue de origen jurisprudencial y se inició en los finales del siglo XIX. La emisión de garantías resulta posible siempre que el banco garante tuviera un interés directo. Por el contrario, la emisión de una garantía a favor de un tercero no fue considerado como actividad bancaria en el caso Bowen vs. Needless National Bank, 94 F. 925, $9^{\text {th }}$ Circuit, 1899; la evolución histórica queda reflejada en GABLE, «Standby Letters of Credit: Nomenclature has confounded analysis», en Law and Policy in International Business, 1980, vol. 12, págs. 914-915; igualmente, CABRILlaC y Moully, Droit des suretes... cit., pág. 366.

11 Las autoridades (controller of the currency) promulgaron diversas reglamentaciones que autorizaban la emisión de standby letters of credit por los bancos estadounidenses. La primera de ellas, de mayo de 1977, fue la Final interpretative Ruling; la segunda, de 1980, impuso condiciones para la validez de los créditos stand-by relativas a las comisiones bancarias, vigencia y monto del crédito, pago contra documentos y reembolso por el ordenante. Los bancos integrados en el Federal Reserve System quedan bajo la Federal Reserve Board, 12 CFR, 1982; el resto de los bancos quedan bajo la Federal Deposit Insurance Corporation, 12 CFR, 1983. Vid. la exposición de CABrillaC y Moully, Droit des suretes, cit., pág. 366.

12 Al respecto, DicKSON y ARABYAn, Le performance bond..., cit., pág. 936; MARTIN, «La lettre de credit stand-by», en Rev. Banque, 1985, n. ${ }^{\circ}$ 6, págs. 31-32.

${ }^{13}$ La expresión pertenece a VASSEUR, en Droit et economie bancaires, fascículo II Les operations de banque, París, 1987-1988, pág. 796-797.

${ }_{14}$ Reglas y Usos Uniformes Relativos a los créditos documentarios, Revisión de 1983, Publicación CCI n. ${ }^{\circ}$ 400, que dio inicialmente cabida a los créditos stand-by en las RUU; Revisión de 1993; Publicación CCI n. ${ }^{\circ}$ 500, Revisión 1993, que mantuvo tal inclusión introduciendo modificaciones primordialmente acerca de los documentos; las RUU se completan con la Publicación CCI, n. ${ }^{\circ}$ 516, The New ICC Standard Documentary Credit Forms; acerca de la evolución seguida por las RUU, puede verse la Publicación CCI n. ${ }^{\circ} 411$, UCP 1974/1983 Revisions Compared and Explained. Documentary credits, y la Publicación CCI, n. ${ }^{\circ}$ 511, Documentary Credits: UCP 500 and 400 Compared.

15 Acerca de la revisión llevada a cabo en 1983, pueden verse BoudinOT, «La revision des regles et usances relatives aux credits documentaires», en Rev. Banque, 1983, págs. 609-612 y págs. 997-1001; EllingER, «The Uniform Customs, their nature and the 1983 Revision», en Lloyd's and Maritime Commercial Law Quaterly, 1984, págs. 578-605; JASINSKY, «Application aux lettres de credit stand-by des regles et usances», en Rev. Banque, 1988, págs. 1116-1120; MARTIN, «La lettre de credit stand-by», en Rev. Banque, 1985, n. 6 , págs. 31-32; especialmente, SCHмITTHOFF, «The New Uniform Customs for Letter of Credit», en Journal of Business Law, 1983, págs. 193 y sigs. 
que los créditos stand-by quedaban incardinados en la figura del crédito documentario ${ }^{16}$. Por el contrario, se aprecia una identidad conceptual entre los créditos stand-by y las garantías independientes, y entre aquéllos y los créditos documentarios ordinarios, de donde puede seguirse la duda acerca de la regulación de las garantías independientes en el marco de la regulación emanada de la CCI, pues sobre la base de tal identidad conceptual, cabría su regulación mediante las RUGD o mediante las RUU. Es de señalar que tal confusión se encuentra, probablemente, en vías de clarificación de resultas de la publicación por la CCI de las International Standby Practices (ISP 98) ${ }^{17}$ que se encuentran culminando su proceso de aceptación por los operadores internacionales. Por ello, procede el análisis de los créditos stand-by al objeto de precisar la regulación de las garantías independientes en el marco de la reglamentación emanada de la $\mathrm{CCI}^{18}$.

\section{La naturaleza y modalidades de los créditos stand-by}

El término stand-by significa un estado de alerta o de espera, es decir, no debiera producirse su participación en la operación e implica la idea de estar preparado para intervenir, de forma que la carta de crédito

16 Acerca de los créditos stand-by, WunNIECKE \& WunNICKE, Standby letters of credit, Business Practice Library, Wiley Law Publications, 1989, en especial, acerca de la jurisprudencia norteamericana, págs. 451-459; Dolan, The law of Letters of Credit (Commercial and standby Credits), Boston $2^{\text {nd }}$. ed. 1990, págs. 7-29, 7-83, 11-28, 11-45, complementada por el suplemento 1995, en CLR, 1985, págs. 1-45; ElLINGER y BARRY, «Standby Letters of credit», en International Business Lawyer, 1978, vol. 6, págs. 604-640; GETZ, «Enjoining the International Standby Letter of Credit: The Iranian Letter of Credit Cases», en Harvard International Law Journal, 1980, págs. 189-252; así como los artículos de KozOLCHYK, «Bank guarantees and letters of credit: time for a return to the fold», en Journal of International Banking Law, 1989, págs. 1-79; KozolCHYK, «The Emerging Law of Standby letters of Credit and Bank Guarantees», en Arizona Law Review, 1980, págs. 319 y sigs; KozOLCHYK, «The financial letter of credit», en Rev. de Droit des Affaires Internationales, n. ${ }^{\circ}$ 4, 1995, pág. 405; LECHIEN, «Les suretés internationales (Etats-Unis d'Amérique 1980-1986)», en Rev. de Droit des Affaires Internationales, n. ${ }^{\circ}$ 5, 1988, págs. 665-686; MATEJCEK, «The law and practice relating to the use of letters of credit and performance bonds in securing contractual performance in Canada and the United States», en Les garanties bancaires dans les contrats internationaux, cit., págs. 49-79; RIGGS, «La lettre de credit standby en tant que garantie bancaire aux Etats-Unis», en Rev. de Droit des Affaires Internationales, n. $^{\circ}$ 3, 1990, págs. 393-400; STUMPF, «Fraud in the transaction: enjoining letters of credit during the iranian revolution», en Harvard Law Review, vol. 93, 1980, págs. 992-1015.

17 Preparadas por The Institute of International Banking Law \& Practice, Publicación CCI, n. ${ }^{\circ} 590$.

18 En la doctrina española, Vicent ChUlia, Compendio crítico de Derecho Mercantil, Barcelona, Bosch, 1986, Tomo II, págs. 423 y sigs.; Alonso UrEBA, «En torno al crédito documentario con particular referencia a las modalidades de créditos transferibles y subsidiarios», en 
stand-by no debería llegar a ser ejecutada si todo se desarrollara normalmente. Asegura el normal desarrollo de una operación comercial o industrial, aunque finalmente pueda resultar siendo ejecutado caso de que se produjeren precisas y determinadas circunstancias ${ }^{19}$. Las cartas de crédito stand-by cubren aspectos diferentes según las operaciones a garantizar, siendo utilizadas en términos generales como garantías comerciales y financieras que revisten una gran versatilidad ${ }^{20}$.

\section{El crédito stand-by financiero}

En virtud del artículo 5-103 $(1, \mathrm{~b})$ del Uniform Commercial Code $(\mathrm{UCC})^{21}$, los bancos americanos pueden emitir cartas de crédito de este

Nuevas entidades, figuras contractuales y garantías en el mercado financiero, Madrid, 1990, págs. 455 y sigs.; TAPIA HERMIDA, «Reglas y usos relativos a los créditos documentarios. Revisión 1983», en Rev. de Derecho Bancario y Bursátil, 1983, págs. 43 y sigs.; HeRNÁNDEZ MARTí, «Créditos documentarios: su cumplimiento y excepciones al mismo», en Estudios Jurídicos Homenaje al profesor Aurelio Menéndez, Tomo III, Cívitas, Madrid, 1996, págs. 3297-3324.

19 Vid. al respecto la exposición de VASSEUR, Les operations de banque cit., pág. 796-797; MARTIN, La lettre de credit stand-by, cit., págs. 31-32; fundamentándose en los dos autores citados, CAPRIOLI, Le credit documentaire: evolution et perspectives, Paría, Litec, 1992, págs. 286-287.

${ }^{20}$ Un cierto porcentaje de tales garantías son libradas a propósito de contratos de construcción o de venta de naves industriales «llave en mano», aun cuando son asimismo utilizadas en relación con situaciones y relaciones jurídicas de gran heterogeneidad En los EEUU, los stand-by son también utilizados en materia civil, de forma que, por ejemplo en el marco del derecho de familia son parte integrante en los acuerdos de custodia de niños, concesión y pagos de pensiones alimenticias, incluso en promesas en matrimonio, y asimismo su utilización se produce en los negocios inmobiliarios. Como ejemplo de la versatilidad de los créditos stand-by, puede hacerse mención del caso de la Bahía de Cochinos, narrado por VASSEUR, Les operations de banque cit., pág. 800. En abril de 1961, la CIA intentó el derrocamiento del régimen de Fidel Castro. Tras el fracaso de la operación de una invasión llevada a cabo por emigrantes cubanos en Bahía de Cochinos, el gobierno del Presidente Kennedy aceptó la permuta de productos farmacéuticos por un valor de cincuenta y tres millones de dólares EEUU a cambio de la puesta en libertad de los prisioneros, operación de difícil conclusión por la desconfianza del régimen cubano, la cual, sin embargo, fue desbloqueada mediante la emisión de una stand-by letter of credit por un banco canadiense contragarantizada por dos bancos estadounidenses, ejecutable mediante la simple declaración del gobierno cubano en el sentido de que los EEUU no habían entregado los productos farmacéuticos.

21 Art. 5-102-1, Uniform Commercial Code, (UCC): «1) This article applies: (a) to a credit issued by a bank if the credit requires a documentary draft or a documentary demand for payment; and (b) to a credit issued by a person other than a bank if the credit requires that the draft or demand for payment be accompanied by a document of title; and (c) to a credit issued by a bank or other person than a bank if the credit is not within subparagraphs a) o b) but conspicuously states that it is a letter of credit or is conspicuously so entitled. El precepto transcrito es complementado por el Art. 5-103 del mismo texto legal (UCC): (a) Credit or letter of credit means an engagement by a bank or other person made at the request of a customer and of a kind within the scope of this Article (section 5-102) that the 
tipo. En la práctica bancaria americana, las cartas de crédito stand-by son igualmente utilizadas como basamento de obligaciones financieras. Las cartas de crédito stand-by emitidas como documentos negociables en los Estados Unidos tienen como finalidad la de actuar como título de garantía para la emisión de efectos negociables. Se permite a las sociedades la emisión de documentos negociables (commercial paper) en el mercado financiero tradicional para obtención de tesorería. El commercial paper corresponde a un pagaré a corto plazo, descontable, emitido al portador o a favor de persona determinada (el comprador). Su vencimiento no suele exceder de nueve meses, aunque la duración normal es inferior a seis meses. Estos pagarés garantizan a sus portadores que serán reembolsados por el garante a su término si el suscriptor no pudiera hacerlo. Son garantías que sirven de sostenimiento a obligaciones financieras de cualquier clase.

La emisión de una carta de crédito stand-by puede tener por objeto una garantía de sumisión (bid bond) que permita garantizar al comprador la conclusión del contrato; o bien, una garantía de devolución de anticipos (advance payment bond) que cubra la devolución de adelantos recibidos si la entrega no llegara a ser efectuada; o bien, una garantía de buena ejecución (performance bond) que conlleve el pago de una suma convenida caso de que el acuerdo no haya sido cumplido; o bien, una garantía de retención (retention money bond) que substituya la retención que hubiere de practicarse por una garantía bancaria. La garantía stand-by podrá también cubrir el mantenimiento de equipamientos (maintenance bonds) o incluso el pago de salarios o de subcontratistas (labour and material payment bond) ${ }^{22}$.

Según la opinión vigente en los EEUU, las ventajas que se derivan de una carta de crédito stand-by residen en la certidumbre y seguridad jurídicas en cuanto a su regulación que se derivan de la adhesión efectuada por los bancos emisores de dichas cartas de crédito a las RUU, por lo que se beneficia de un conjunto de normas jurídicas de derecho uniforme y universal; por otra parte, ayudan al mantenimiento del equilibrio contractual entre las partes, pues la carta de crédito stand-by sirve de contrapeso a la garantía de pago probablemente emitida por el comprador a favor del vendedor. A este respecto, constituyen, al igual

issuer will honor drafts or other demand for payment upon compliance with the conditions specified in the credit. A credit may be either an agreement to honor or a statement that the bank or other person is authorised to honor. (b) A documentary draft or a documentary demand for payment is one honor of which is conditioned upon the presentation of a document or documents. «Document» means any paper including document of title, security, invoice, notice of default and the like». Vid. sobre la cuestión, RIGGS, L'utilisation des lettres de credit a l'appui d'emissions d'effets negociables aux Etats-Unis, cit., págs. 806 y 819.

22 CAPRIOLI, Le credit documentaire... cit., págs. 292-293. 
que una garantía independiente, la contrapartida de un crédito documentario ordinario. La carta de crédito stand-by emitida en favor del comprador equilibra la garantía de pago (que podría ser un crédito documentario). En el contexto de esta operación, se invierte completamente el proceso del crédito documentario ordinario o clásico, pues el beneficiario es el comprador mientras que el librador es el vendedor ${ }^{23}$.

\section{La utilización del crédito stand-by en garantía del pago del precio}

Cuando se utiliza un crédito stand-by como garantía del pago adeudado derivado de un contrato de compraventa, aquél no tiene como finalidad servir de garantía de la ejecución de un contrato. Ello no impide la apreciación de acentuadas similitudes entre el crédito stand-by comercial y el crédito documentario ordinario. Al igual que en éste, la emisión de una carta de crédito stand-by comercial procede del comprador, que es su ordenante, quien solicita su emisión en favor del vendedor, que resulta ser su beneficiario. El uso del calificativo «comercial» en la carta de crédito stand-by emitida para garantizar una operación de importación es consecuencia de su utilización como garantía del pago de una compraventa sin que pueda ser considerada como un medio de pago.

Asimismo, puede señalarse la similitud consistente en que el importe garantizado alcanza a la totalidad de la operación y no a una parte de la misma. En consecuencia, además de las similitudes de índole formal entre el crédito stand-by comercial y el crédito documentario ordinario, aquél muestra una única diferencia con éste, pues no constituye un medio de pago normal, sino que lo es por defecto ${ }^{24}$, de forma que el banco no tendrá que pagar su importe a no ser que el comprador no pague, es decir, no cumpla su prestación correspondiente en la operación subyacente. El pago de la carta de crédito es secundario o subsidiario, puesto que sanciona una falta de pago $^{25}$. Ambas modalidades de cartas

23 BoudinOt, La revision des regles et usances relatives aux credits documentaires, cit., págs. 609-612 y págs. 997-1001.

$24 \mathrm{Al}$ respecto, cfr. la exposición de PUECH, Credit documentaire, garanties bancaires, stand-by letters of credit... cit., pág. 19.

25 El desarrollo de la carta de crédito stand-by comercial ha supuesto algunos problemas para los bancos, tales como, de un lado, la posibilidad de que pueda tener lugar un doble pago y, de otro, el hecho de que los documentos no otorgan ningún derecho sobre la mercancía, así como la apreciación de la calidad y de la capacidad del ordenante. En cuanto al primer punto, para paliar el riesgo del doble pago, se ha adoptado un modelo de carta de crédito stand-by como pago de mercancías en el cual el beneficiario certificará no haber recibido el pago. Considerando que el banco no tiene necesidad de documentos originales, la última dificultad depende de la apreciación de los riesgos por el propio banco caso a caso. Cfr. CAPRIOLI, Le credit documentaire... cit., pág. 296. 
de crédito stand-by son instrumentos de garantía, por lo que podrían quedar sin surtir sus efectos si en un crédito stand-by financiero el exportador ejecutara normalmente sus obligaciones, o si en un crédito stand-by comercial el comprador pagara el importe de la transacción.

\section{La diferente naturaleza jurídica de los créditos documentarios ordinarios y de los créditos stand-by}

El funcionamiento de la carta de crédito stand-by es similar al de la garantía independiente en cuanto al fondo, y es similar al del crédito documentario en cuanto a la forma. Sin embargo, los principios que lo inspiraron preveían su integración en la técnica del crédito documentario dado que la práctica bancaria buscaba insertar los stand-by en la regulación general de los créditos documentarios. Las discusiones han sido abundantes respecto a su inclusión en la Revisión de las RUU de 1983, en razón de este carácter híbrido ${ }^{26}$.

Para un sector doctrinal ${ }^{27}$, las semejanzas apreciables entre la carta de crédito stand-by y el crédito documentario ordinario resultan de peso suficiente como para concluir la identidad de naturaleza jurídica entre ambos instrumentos ${ }^{28}$.

26 Antes de dicha revisión, era práctica frecuente la referencia a la aplicación de las RUU en las cartas de crédito stand-by con la intención de garantizar las obligaciones de suministros o de prestaciones de los servicios a la exportación. Vid. al respecto MARTIN, $L a$ lettre de credit standd-by cit., págs. 31-32.

27 Tal línea doctrina está representada principalmente, aun cuando con matizaciones, por Boudinot, La revision des regles et usances relatives aux credits documentaires, cit., págs. 609-612 y págs. 997-1001; JASINSKY, Application aux lettres de credit stand-by des regles et usances, cit., págs. 1010-1014; MARTIN, La lettre de credit stand-by, cit., págs. 31-32; abiertamente, CAPRIOLI, Le credit documentaire... cit., especialmente pág. 287. Tales semejanzas permiten concluir la específica particularidad de la carta de crédito stand-by, pues las operaciones de crédito stand-by y los créditos documentarios son compromisos bancarios crediticios Por otra parte, ambos mecanismos implican estructuras en los planos jurídico y financiero en los que son similares las personas comprometidas, comparten la misma independencia frente al contrato subyacente y deben cumplir con determinados requisitos en orden a la ejecución del compromiso del banco. Asimismo, cabe resaltar la filiación jurídica que preside los destinos del crédito documentario y de la carta de crédito stand-by en cuanto garantías bancarias internacionales. Para dicho sector doctrinal, se podría, pues, deducir que existe una identidad de técnica bancaria y de naturaleza jurídica entre el crédito documentario y la carta de crédito stand-by.

28 Los créditos stand-by y los créditos documentarios ordinarios son, por su propia naturaleza, compromisos bancarios crediticios Es la consideración generalmente deparada por la doctrina francesa. El crédito documentario es incardinable entre los créditos a la importación, pues el banco prestará su firma al comprador para garantizar la realización de la prestación financiera y pagará al vendedor a partir del momento en que éste haya presentado los documentos previstos en el momento de la apertura del crédito. En consecuencia, 
Por otra parte, se pone de manifiesto que, en la práctica, las relaciones económicas internacionales que conllevan la emisión de una garantía implican la participación de una misma estructura personal cuyos elementos resultan ser: un ordenante, un banco emisor que asume un compromiso de pago, un beneficiario que debe dar normalmente cumplimiento a determinadas condiciones y, a veces, un segundo banco intermediario; todo ello con independencia del concreto instrumento utilizado, fuere éste tanto un crédito documentario ordinario como una carta de crédito stand-by, y todo ello, asimismo, basado en la aportación de los documentos cuya entrega ha sido pactada por las partes.

La apreciación de la mencionada estructura operacional lleva a presumir que ello habría sido la causa de su inclusión en las RUU, por lo que la identidad de naturaleza entre los créditos documentarios ordinarios y los créditos stand-by se fundamentaría en el art. 2 de las RUU ${ }^{29}$, que contiene la definición de crédito documentario y que abarca tanto a los créditos documentarios ordinarios como a las cartas de crédito stand-by, para de ello deducir una identidad de naturaleza entre ambos mecanismos $^{30}$. En apoyo de lo anterior, se $\operatorname{arguye}^{31}$ que las considera-

sus características responden a la definición de crédito pues éstos son compromisos asumidos por un banco en favor de terceros a petición de un cliente. Al respecto, Bonneau, Droit Bancaire, París, Montchrestien, 1994, pág. 391; Rives LANGE y ConTAMYNE RaYNAUd, Droit Bancaire, 6. ${ }^{\mathrm{a}}$ edición, París, Precis Dalloz, 1995, pág. 721; SiMLER, Le cautionnement... cit., n. ${ }^{\circ} 869$; Gavalda y StoufFlet, Droit du credit, Tomo I, n. ${ }^{\circ} 89$.

${ }^{29}$ RUU, Publicación CCI, n. ${ }^{\circ}$ 500: el art. 1 de las RUU establece que «Las presentes Reglas y Usos Uniformes...son de aplicación a todos los créditos documentarios (incluyendo las cartas de crédito stand-by en la medida en que sea aplicable...). Por su parte, el art. 2 de las RUU define el crédito documentario en el sentido de que lo es «todo acuerdo, cualquiera que sea su denominación o descripción, por el que un banco, obrando a petición y de conformidad con las instrucciones de un cliente (ordenante) o en su propio nombre: I) se obliga a hacer un pago a un tercero (beneficiario) o a su orden, o a aceptar y pagar letras de cambio libradas por el beneficiario; II) o autoriza a otro banco para que efectúe el pago o para que acepte y pague tales instrumentos de giro; III) o autoriza a otro banco para que negocie contra la entrega de los documentos, siempre y cuando se cumplan los términos y las condiciones del crédito».

30 Entre otros autores, BoudinOT, La revision des regles et usances relatives aux credits documentaires, cit., págs. 609-612 y págs. 997-1001; JASINSKY, Application aux lettres de credit stand-by des regles et usances, cit., págs. 1116-1120; MARTIN, La lettre de credit stand-by, cit., págs. 31-32; CAPRIOLI, Le credit documentaire... cit., págs. 274 y sigs. Hay un salto lógico en el razonamiento seguido por el citado autor, para cuya excusa aduce que la función de la CCI es conseguir la unificación de las prácticas bancarias y no su división. En realidad, esta orientación doctrinal buscaba más defender la labor unificadora de la CCI y extender la regulación contenida en las RUU a los créditos stand-by, en busca de seguridad jurídica, que defender estrictamente la identidad de naturaleza jurídica entre éstos y los créditos documentarios ordinarios.

31 Cfr. CAPRIoli, Le credit documentaire... cit., pág. 280. 
ciones hechas por la Comisión Bancaria de la CCI ${ }^{32}$ acerca del citado precepto ponen de relieve una substancial identidad en la naturaleza jurídica de los compromisos.

En realidad, la preocupación de la CCI estribó en la consecución de la mayor unificación posible en cuanto a la práctica de los mecanismos bancarios de garantía, entre los cuales la regulación relativa a los créditos documentarios ostentaba un reconocimiento universal ${ }^{33}$. La denominación créditos documentarios stand-by, propagada desde los EEUU, y la consideración analógica dispensada a los mismos en relación a los créditos documentarios ordinarios llevaron a la inclusión de los créditos stand-by en la regulación de los créditos documentarios en aras a la evitación de la diversificación jurídica. Realmente hubiera sido sorprendente que la CCI hubiera dado una definición diferente a los créditos stand-by cuando su propósito era el de buscar su sumisión a una misma regulación. La aceptación por las RUU de un nuevo tipo distinto de crédito documentario, reconociendo la existencia de un mecanismo distinto del crédito documentario ordinario, hubiera sido actitud poco favorable para la unidad de la institución ${ }^{34}$.

En efecto, cabe apreciar un paralelismo entre ambas figuras, crédito documentario ordinario y carta de crédito stand-by, pues ambas son compromisos de pago independientes del contrato subyacente, cuya realización está sometida al respeto de las condiciones documentarias estipuladas en el crédito. La independencia de la obligación del banco en la carta de crédito stand-by caracteriza igualmente al crédito documentario ordinario. El objeto principal de la carta de crédito stand-by es el de garantizar a primera demanda un pago al beneficiario caso de

32 Vid. Doc. CCI. n. ${ }^{\circ}$ 470/367, de 4 de agosto de 1980, así como la Opinión (Avis) (1980-1981) al respecto de la Comisión de Práctica y Técnica Bancarias de la CCI. Cfr. CAPRIOLI, Le credit documentaire... cit., pág. 282, notas 36 y 37.

${ }^{33}$ Comentarios acerca de la cuestión de WheBLe, Doc. CCI. n. ${ }^{\circ} 470 / 561$, de 11 de octubre de 1988, y Doc. CCI. n. ${ }^{\circ}$ 470/Int. 248, de 13 de diciembre de 1988, en sus informes dirigidos a los miembros de la Comisión Bancaria en los que el autor propone la supresión de las stand-by letters of credit del texto de las RUU «car cela s'applique a des situations par defaut. La question será couverte par les nouvelles regles sur les garanties».

${ }^{34}$ Otros autores señalan alguna otra similitud, como la que ambas muestran en cuanto a su función compartida de ser garantías bancarias de pago, bien lo fueren del pago de un suministro de mercancías, bien lo fueren del buen fin de una prestación contractual o del pago de su incumplimiento. Ambas deben ser objeto de notificación al beneficiario por su emisor a fin de tener un valor frente a terceros. La conjugación de estas convergencias ha producido una nueva garantía llamada carta de crédito stand-by, que es en realidad una garantía bancaria independiente emitida en forma de crédito documentario. BonTOux, Les garanties bancaires dans le commerce international, cit., págs. 171-174; en el mismo sentido, Puech, «Les garanties bancaires dans le commerce international», en Actualites BFCE, mayo 1984, págs. 3-4. 
incumplimiento de sus prestaciones contractuales por el deudor. Ya en 1979, en su XII Sesión, la Uncitral ${ }^{35}$ había calificado y definido las cartas de crédito stand-by de igual forma en su informe, afirmando que «la carta de crédito emitida por un banco en favor del beneficiario establece entre el banco y el beneficiario una relación jurídica independiente de este contrato subyacente... De conformidad con los términos convenidos en el contrato subyacente especificará las condiciones que debe cumplir el beneficiario para tener derecho a reclamar el pago... el banco está obligado a pagarle, sin tener en cuenta diferencias que podrían existir entre el beneficiario y el librador en lo que concierne al contrato (subyacente) ${ }^{36}$.

Sin embargo, junto a las similitudes apuntadas, más aparentes que reales, cabe la apreciación de diferencias de índole básica que apuntan a una diferenciación en cuanto a la naturaleza jurídica de ambos instrumentos. En el aspecto económico, las funciones de ambos mecanismos parecen ser opuestas, puesto que la carta de crédito stand-by es una garantía mientras que el crédito documentario es un medio de pago. Por otro lado, el crédito stand-by se presenta bajo la forma y la apariencia de un crédito documentario ordinario, aunque el formalismo empleado esté limitado ${ }^{37}$.

Los créditos documentarios se incardinan en las «operaciones de mediación en los pagos» dentro de la tipología de las operaciones bancarias $^{38}$. La utilización del término «crédito» puede ser ajena a los perfiles de la figura contemplada, pues el pago por el banco al vendedor no implica necesariamente una relación de crédito entre banco y comprador, dado que el banco puede disponer de fondos del propio comprador (v.gr., en depósito o en cuenta corriente). A su vez, en la relación

35 Cfr. Doc. A/CN.9/163, de 9 de mayo de 1979, titulado Stand-by letters of credit.

36 Cfr. Ellinger, «Letters of credit», en The Transnational Law of International Commercial Transactions, cit., pág. 248. Dada la identidad de naturaleza jurídica, los tribunales suelen estimar que su régimen jurídico es también el mismo. Bajo esta perspectiva, el estudio de la jurisprudencia anglosajona ha llevado al citado autor a concluir que «cases concerning stand-by credits can, however, be of relevance in respect of issues concerning documentary credits as the courts are inclined to apply a uniform body of law to both types of letter of credit».

37 A diferencia de los créditos documentarios ordinarios o comerciales, las cartas de crédito stand-by son, en principio, operaciones bancarias ligadas a la exportación, que contienen un compromiso de pago al comprador caso de incumplimiento de sus obligaciones por el vendedor. La carta de crédito stand-by se sitúa, bien entre los compromisos a la importación a título de garantía para la compra de mercancías, bien entre los compromisos a la exportación como garantía de la correcta ejecución del contrato. PUECH, «Les stand-by letters of credit: une force meconnue», en Actualites BFCE, octubre 1986, págs. 1-2.

38 Vicent Chulia, Compendio crítico..., cit., II, pág. 423. 
entre el banco y el vendedor, no hay crédito si el pago es al contado, si bien cabe que la modalidad de pago consista en la aceptación de una letra por el comprador que descuenta a su vez el banco al vendedor, originándose entonces una relación de crédito. Todo ello pone de manifiesto que puede haber crédito, pero que ello es accesorio respecto de lo esencial que resulta ser la mediación en los $\operatorname{pagos}^{39}$. En cualquier caso, parece claro que tanto los créditos documentarios ordinarios, los créditos stand-by y las garantías independientes son reconducibles conjuntamente a tal renglón de actividad bancaria, fuere ésta de índole crediticia o de mediación en los pagos según las distintas concepciones.

\section{Las diferentes finalidades económicas}

Puede apreciarse la existencia de una diferencia relativa a la dualidad funcional del crédito documentario, que es simultáneamente instrumento de garantía y medio de pago, frente al monismo funcional que preside el funcionamiento de la carta de crédito stand-by, que es únicamente garantía de ejecución.

Por otra parte, existe diferencia en cuanto a las finalidades económicas de ambos instrumentos, pues uno de ellos está destinado a su ejecución y el otro, al menos en principio, a no ser ejecutado. La función del crédito documentario consiste en asegurar a su beneficiario el pago de una mercancía o de una prestación de servicios por lo que su técnica implica obligatoriamente su realización, de forma que, si todo se desarrolla normalmente y de conformidad con el contrato, aquél es ejecu$\operatorname{tado}^{40}$. Por su parte, la carta de crédito stand-by sanciona la inejecución o la defectuosa ejecución del contrato subyacente. Si las partes respetan los términos del contrato, no será ejecutada. Ambos mecanismos sirven, pues, necesidades económicas diferentes ${ }^{41}$.

\section{El diferente grado de formalismo}

Un crédito documentario ordinario suele hacer referencia a un conjunto de documentos de índole comercial cuya presentación dará lugar a la realización del crédito documentario de manera automática. Tales

39 Alonso Ureba, En torno al crédito documentario..., cit., pág. 457.

40 Bonneau, Droit Bancaire..., cit., pág. 385.

${ }^{41}$ En cuanto a su coste, señala CAPRIOLI, Le credit documentaire..., cit., pág. 288, que los bancos aplican a los créditos stand-by la misma comisión que a las garantías independientes en razón del mayor riesgo financiero, aunque el riesgo técnico relativo al examen de los documentos tiende a reducirse. La idea de un riesgo mínimo en el examen de los documentos conduce a considerar el formalismo que exhibe, en ambos casos, exigencia idéntica. 
documentos tienen por misión probar o aportar la presunción de que el contrato subyacente ha recibido correcta ejecución, estableciendo el normal cumplimiento de sus obligaciones por parte del vendedor, de manera que, al dar cumplimiento final a sus obligaciones, el beneficiario tiene la certeza de ser pagado.

En cambio, la ejecución de una carta de crédito stand-by contra documentos puede requerir un único documento, cual es la simple declaración del beneficiario de la carta de crédito stand-by. La declaración mencionará que no ha recibido la prestación o que la prestación ha sido mal ejecutada. A veces, la declaración del beneficiario, que afirma que el reembolso no ha sido hecho o que el resultado no ha sido alcanzado, se acompaña de otros documentos librados por terceras personas (peritos, ingenieros, asesores, árbitros, etc.). El carácter documentario de las cartas de crédito stand-by reviste sin embargo particular importancia, puesto que la obligación de presentación de los documentos permite diferenciarlas de las garantías independientes que pueden ser simples, supuesto en el cual la reclamación del pago por el beneficiario no debe ir acompañada de documento alguno ${ }^{42}$.

Cabe apreciar una notoria diferencia entre el acusado formalismo que reviste el crédito documentario cuando se utiliza para el pago del precio de una compraventa de mercancías, supuesto en el que se requiere un conjunto completo de documentos que representan la prueba del cumplimiento de las obligaciones del vendedor, frente al formalismo simplificado en el supuesto de un crédito stand-by. Ello desvirtúa el general carácter documentario inicialmente previsto por las RUU, puesto que el mecanismo implica la idea de que los documentos representan las mercancías. En las cartas de crédito stand-by, las exigencias documentarias se ven reducidas a su más simple expresión para satisfacer condiciones de forma mínimamente necesarias para poder quedar incorporadas al mecanismo del crédito documentario ${ }^{43}$.

Por otra parte, el significado de la documentación exigida en la carta de crédito stand-by es distinto del que se aprecia en el crédito documentario ordinario o clásico, pues los documentos no intervienen en el mismo momento de la operación. En este último, se emplean como contrapartida de un compromiso bancario, mientras en aquél fundamentan la sanción de una inejecución contractual. En el crédito stand-by, los documentos emanan esencialmente del beneficiario; por

42 Martin, art. cit., págs. 25-27. Parece claro el escaso valor del argumento que lleva a concluir que un crédito stand-by es una garantía independiente documentaria.

43 JASINSKY, Application aux lettres de credit stand-by des regles et usances, cit., págs. 1116-1120. 
el contrario, los documentos emanados de terceros pueden ser numerosos en el caso del crédito documentario. Esta diferencia se explica porque los documentos exigidos en un crédito documentario deben corresponderse exactamente con las necesidades de la operación comercial. Fines económicos opuestos entrañan un sentido y un alcance diferente en el plano de las obligaciones documentarias que emanan del contrato.

La finalidad económica del crédito documentario y las condiciones documentarias para la realización del crédito son diferentes de las exigidas en las cartas de crédito stand-by. Por tanto, una transposición de las RUU a los créditos stand-by podría resultar distorsionadora.

Por último, existe una dualidad de cartas de crédito stand-by, pues, cuando el banco emite una carta de crédito stand-by a solicitud del importador, tal carta de crédito resulta comparable con un crédito documentario clásico, en tanto que la emisión de una carta de crédito stand-by a solicitud del exportador la hace comparable a una garantía independiente.

Las similitudes que se tratan de resaltar entre los créditos documentarios ordinarios y los créditos stand-by, de modo que quepa la subsunción de éstos en la general noción de crédito documentario, con el consiguiente corolario de su procedente sumisión a las RUU, no parecen encontrarse suficientemente respaldadas. Por el contrario, las diferencias apreciables entre ambas figuras, particularmente las relativas a la función económica y jurídica por ellas desempeñada, conducen al establecimiento de una clara diferenciación entre créditos documentarios ordinarios y créditos stand-by, así como a la apreciación de la plena similitud de éstos con las garantías independientes ${ }^{44}$. En tal línea de pensamiento, en su comentario a propósito de la Revisión de 1983 de las RUU aplicables a los créditos documentarios, SchмiтtнofF ${ }^{45}$ afirma que los «créditos stand-by son esencialmente garantías bancarias independientes, de forma que la obligación de pago del banco nacerá si el deudor principal, comprador o suministrador según fuere el caso, incumple sus obligaciones, aunque la obligación del banco dependerá del cumplimiento de determinadas condiciones, tales como la presentación de los documentos requeridos. La carta de crédito stand-by es una invención americana. En los EEUU ciertos bancos tienen prohibida la

44 Tal es el criterio en que se fundamenta la Convención de la Uncitral en materia de garantías independientes, que viene a refundir en cuanto a su regulación a los créditos stand-by y a las garantías autónomas o a primera demanda.

${ }^{45}$ Cfr. Schmitthoff, The New Uniform Customs for Letter of Credit, cit., págs. 193 y sigs. 
emisión de garantías bancarias independientes. Evaden tal prohibición mediante la emisión de cartas de crédito stand-by, particularmente en el mercado interno». Prosigue el citado autor afirmando que «las cartas de crédito stand-by son una institución tan diferente de los créditos documentarios ordinarios que resulta inapropiado y susceptible de generar confusión el que las nuevas RUU se extiendan a dicha clase de instrumento financiero».

Realmente, los créditos documentarios ordinarios son algo distinto de las cartas de crédito stand-by, a veces denominadas «créditos documentarios de garantía», que, de acuerdo con Alonso UREBA ${ }^{46}$, «no sirven a una finalidad de mediación en el pago, sino de garantía de una suma de dinero debida por el ordenante del crédito documentario al beneficiario, generalizándose hoy como garantía bancaria y aproximándose en este sentido a las llamadas «garantías abstractas o a primer requerimiento», si bien, a diferencia de éstas, el pago por el banco puede condicionarse a la entrega de ciertos documentos». El citado autor afirma $^{47}$ a continuación, que su inclusión en las RUU bajo la denominación de stand-by letters of credit puede inducir, aún más si cabe, a cierta confusión. 48

Tal parece ser, igualmente, la opinión de la propia CCI, pues, aun cuando mantenga la simultánea vigencia de diferentes regulaciones sobre los créditos documentarios y sobre las garantías a primera demanda, la Introducción de las RUGD afirma que los stand-by credits are technically within the definition of a demand guarante $e^{49}$.

46 Cfr. Alonso UReBA, En torno al crédito documentario con particular referencia a las modalidades de créditos transferibles y subsidiarios, cit., pág. 457. Cita, a su vez, a GutTerdidge y Megrah, The Law of Bankers' Commercial Credits, pág. 9.

47 Alonso Ureba, ibidem.

48 En la misma línea de opinión, Ventris, Bankers' Documentary credits, 3. a edición, London Press Ltd., 1990, pág. 148, quien afirma que «In this respect many may consider the inclusion in the code of bankers' guarantees in the guise of stand-by letters of credit a gross error, and wich may well turn out to be a «wolf in sheep's clothing». Bankers' documentary credits and stand-by letters of credit have nothing in common. Indeed, the one is the antithesis of the other». En el mismo sentido se pronuncia Coleman, Performance guarantees, cit., pág. 229. Igualmente, ElLINGER, «Stand-by letters of credit», en International Business Lawyer, 1978, vol. 6, pág. 622; asimismo, BERTRAMs, op. cit., pág. 6, en donde el autor se pronuncia con rotundidad afirmando que considerar como diferentes a los créditos stand-by y a las garantías independientes europeas «is a fallacy». Igualmente, pueden verse los comentarios acerca de la cuestión de WHEBLE, Doc. CCI. n. ${ }^{\circ} 470 / 561$, de 11 de octubre de 1988, y Doc. CCI. n. ${ }^{\text {o } 470 / I n t . ~ 248, ~ d e ~} 13$ de diciembre de 1988, informes dirigidos a los miembros de la Comisión Bancaria, en los que el autor propone la supresión de los stand-by letters of credit del texto de las RUU «car cela s'applique a des situations par defaut. La question sera couverte par les nouvelles regles sur les garanties».

49 RUGD, Publicación CCI, n. ${ }^{\circ}$ 458, pág. 1. 


\section{La aplicabilidad de las RUU a los créditos stand-by}

Los créditos stand-by están sujetos a las RUU desde la Revisión de 1983. Sin embargo, de acuerdo con lo anteriormente expuesto, constituyen una figura o mecanismo jurídico que, según la modalidad de que se trate, puede corresponderse con la noción de crédito documentario ordinario, lo que sería el caso del crédito stand-by comercial; por el contrario, puede mostrar substanciales diferencias conceptuales frente a la figura del crédito documentario, lo que sería el caso del crédito stand-by financiero del que, incluso, podría llegarse a afirmar que su sometimiento a las RUU tiene algo de artificial, probablemente forzado por el interés de los bancos americanos en conseguir tal equiparación y sometimiento como medio para eludir prohibiciones contenidas en el propio derecho norteamericano respecto de las actividades que los bancos americanos podrían desarrollar o tener vetadas.

A pesar de ello, para un sector doctrinal, la emisión de una garantía independiente en forma de crédito documentario sometido a las RUU ofrece la ventaja de la seguridad jurídica apreciable en la figura del crédito documentario ${ }^{50}$. La utilización de la forma de un crédito stand-by y su sumisión a las RUU hará que se beneficie de las ventajas que resultan de ser unas reglas universalmente aceptadas, lo que se contrapone al hecho de que las reglas reguladoras de las garantías independientes no poseen un carácter consuetudinario, mientras que las RUU son internacionalmente consideradas como un conjunto de reglas consuetudinarias universales, con larga tradición en la práctica bancaria.

Por otro lado, el carácter documentario de los créditos stand-by les otorga una consistente fuerza jurídica, de forma que el ordenante sabrá siempre, de forma precisa, las razones que llevan al beneficiario a la reclamación del pago. Por el contrario, en el supuesto de una garantía independiente no sometida a las RUU, la declaración del beneficiario en el sentido de que el ordenante no ha dado completo cumplimiento a la ejecución de sus prestaciones contractuales será la prueba formal para la reclamación de la garantía, permitiendo, por tanto, una reclamación abusiva de aquella. La responsabilidad del beneficiario podrá ser más fácilmente establecida en orden a la recuperación de lo indebidamente percibido y, asimismo, se pueden soslayar los inconvenientes derivados de la ambigüedad de la noción de abuso manifiesto puesta de manifiesto por la jurisprudencia de diferentes países en materia de ga-

50 CAPRIOLI, Le credit documentaire... cit., pág. 293, y los autores citados en notas 61 y 62 . 
rantías independientes. En suma, para un sector doctrinal ${ }^{51}$, a pesar de las imperfecciones de que todavía se resiente la regulación relativa a los créditos documentarios, el crédito stand-by regulado por las RUU es visto como instrumento más seguro que las garantías a primer requerimiento.

Probablemente, tal punto de vista no puede ser compartido en la actualidad, al menos desde el punto de vista de la práctica europea. La figura de los créditos stand-by es creación americana, artificialmente forzada por las particularidades de la legislación bancaria de los EEUU y artificialmente introducida en las RUU, de donde se sigue incluso una defectuosa aplicabilidad de las RUU. Conceptualmente, no son cosa distinta de las garantías independientes, de donde se sigue una cierta desvirtuación de la noción de crédito documentario. Por el contrario, las garantías independientes disponen hoy de una adecuada regulación en las RUGD, aun cuando no se encuentre tan decantada como la contenida en las RUU. Por último, la Convención de la Uncitral, que refunde y contempla ambas figuras de garantía, significará una mayor uniformidad en la práctica universal.

Producida la inclusión de los créditos stand-by en las RUU, la consecuencia estriba en su sometimiento a las previsiones en aquellas contenidas. Sin embargo, las particularidades que los créditos stand-by ponen de manifiesto, su diferencia conceptual con la normalmente aceptada como noción de crédito documentario, su marcada proximidad a la noción de garantía independiente, todo ello trae como consecuencia la incompleta adecuación de las RUU para la total regulación de los créditos stand-by y, en consecuencia, la exclusión de determinados preceptos de las RUU en su aplicación a los créditos stand-by. No hay pautas en las RUU ${ }^{52}$ o en otros pronunciamientos de la CCI que permitan la precisión de los artículos que resultan de aplicación al crédito stand-by Por el contrario, determinados preceptos han sido declarados como inaplicables.

51 BoudinOt, La revision des regles et usances relatives aux credits documentaires, cit., págs. 609-612 y págs. 997-1001; CAPRIOLI, Le credit documentaire... cit., pág. 298 JASINSKY, Application aux lettres de credit stand-by des regles et usances, cit., págs. 11161120; MARTIN, La lettre de credit stand-by, cit., págs. 31-32.

${ }^{52}$ Cfr. la exposición de Cabrillac y Moully, Droit de suretés cit., pág. 367, quienes ponen de manifiesto que la CCI ha afirmado que las cartas de crédito stand-by pueden encajar en la definición de los créditos documentarios, de acuerdo con lo dispuesto en las Disposiciones generales, y que deben contener una referencia expresa a las RUU. Vid. asimismo las decisiones de la ICC Banking Commission on queries relating to Uniform Customs and Practice for Documentary Credits, Publicación CCI n. ${ }^{\circ} 371$, 1980, pág. 11 . 


\section{Los preceptos de las RUU aplicables a los créditos stand-by}

El art. 1 establece la aplicabilidad de las RUU a los créditos standby, pero sugiere que no lo son en su totalidad, ni marca las pautas para tal discernimiento.

«Las presentes Reglas y Usos Uniformes...son de aplicación a todos los créditos documentarios (incluyendo las Cartas de Crédito Stand-by, en la medida en que sea aplicable)...»

Entre ellos se encuentran:

1. ${ }^{\circ} \mathrm{El}$ art. 2 recoge la definición de crédito documentario y contempla los requisitos que permiten la calificación de un crédito documentario como ordinario o comercial, o bien como crédito documentario stand-by ${ }^{53}$;

2. ${ }^{\circ}$ Los arts. 3 a 6 expresan el carácter abstracto del compromiso bancario, significando la plena independencia del crédito frente al contrato subyacente, lo sea éste de compraventa o de cualquier otra naturaleza. El art. 3 matiza que tal independencia se mantendrá aun cuando en el crédito se incluyere alguna referencia al mencionado contrato, lo que parece una precisión dedicada a los créditos stand-by que suelen contener alguna referencia al contrato subyacente;

3..$^{\circ} \mathrm{El}$ art. 4 establece que las partes deben tomar únicamente en consideración los documentos y no las mercancías, junto a lo cual se alude a la extensión de las RUU a la prestación de servicios u otras prestaciones, mención que resulta de carácter básico aun cuando novedosa en el texto de las RUU, y que asimismo parece dedicada a los créditos stand-by que son emitidos en relación a otras varias clases de relaciones jurídicas más allá de la compraventa de mercancías;

4. ${ }^{\circ}$ El art. 5 se refiere a la emisión de los créditos y a las modificaciones que pudieran ser introducidas, precisando que deberán ser completas y precisas, aun cuando la simple declaración acerca de la inejecución del contrato mencionado en la carta de crédito será suficiente a los efectos del mencionado precepto;

5. ${ }^{\circ}$ La notificación de la carta de crédito stand-by tendrá lugar de acuerdo con las previsiones del art. 8, mientras que los arts. 10 y 11 regulan lo relativo al alcance de la obligación asumida por

53 Cfr. supra, nota n. ${ }^{\circ} 14$. 
el banco, fijando los requisitos para la notificación y la confirmación cualquiera que fuere el tipo de crédito emitido;

6. ${ }^{\circ}$ Pueden resultar igualmente de aplicación los arts. 15 a 21 relativos a las responsabilidades de los bancos que deberán examinar los documentos en plazo y con cuidado razonables, así como su apariencia de conformidad con las condiciones del crédito, debiendo además resultar compatibles entre sí;

7. ${ }^{\circ}$ En lo relativo a los documentos, la redacción dada a los arts. 22 a 24 es consecuencia de la inclusión de los créditos stand-by por lo que resultan de aplicación a éstos;

8. ${ }^{\circ}$ Los arts. 25 a 42, relativos a los documentos de transporte, no pueden resultar de aplicación a los stand-by financieros pero sí a los comerciales;

9. ${ }^{\circ}$ Otras disposiciones de las RUU tampoco suscitan dudas en cuanto a su posible aplicación. Así los arts. 44 y 45 son muestra de la adaptación de la Revisión de 1983 a la oficialización de los stand-by. El art. 46 contiene la noción de fecha última válida para la presentación de los documentos de acuerdo con las previsiones del crédito. Asimismo, tampoco presentan problemas los arts. 48 y 49, ni los arts. 51 a 53 que precisan el significado de determinados términos y expresiones utilizadas en las relaciones comerciales.

\section{Los preceptos de las RUU no aplicables a los créditos stand-by}

Por el contrario, parece claro que no resultan de aplicación a la regulación de los stand-by las disposiciones de las RUU relativas a la irrevocabilidad del crédito, las relativas a los plazos para la presentación de los documentos, así como las disposiciones que contemplan la transferencia del crédito ${ }^{54}$.

Los arts. 7 a 9 de las RUU se refieren a la naturaleza del crédito. Entre sus previsiones se encuentra la necesaria mención del carácter de irrevocabilidad sin la cual el crédito será considerado como revocable y, por lo tanto, podría ser alterado o anulado. En la práctica, no son frecuentes los créditos revocables porque la revocabilidad sería, desde un principio, contraria a la función de garantía de pago o indemnización consubstancial a la figura. La irrevocabilidad aparece como intrínseca a la figura, y sería difícil imaginar la hipótesis en la cual el beneficiario solicitara la emisión de un crédito stand-by revocable para garantizar el

54 Vid. el análisis de CAPRIOLI, Le credit documentaire... cit., págs. 298-307. 
pago de unas mercancías o la indemnización correspondiente a la inejecución de una prestación contractual. Se trata, en suma, de previsiones opuestas al espíritu de los créditos stand-by.

El art. 47 RUU regula el plazo de presentación de los documentos, en virtud del cual, a falta de estipulación expresa, regirá el plazo de veintiún días para dicha presentación, de forma que, transcurrido dicho plazo, el banco se encontrará en la tesitura de poder negarse a la aceptación de aquellos documentos cuya presentación hubiere incurrido en retraso ${ }^{55}$. Dicha previsión no se aplica a los créditos stand-by, como ha declarado la Comisión Bancaria de la CCI a propósito del art. 47 RUU ${ }^{56}$. En efecto, un crédito stand-by será de utilidad caso de inejecución de alguna o algunas prestaciones contractuales. La función de garantía inherente a este tipo de crédito tendrá como consecuencia la presentación de cualquier documento tras la expiración del mencionado plazo, de donde se sigue la inaplicabilidad del mencionado precepto a los créditos stand-by.

El art. 54 RUU se refiere a las circunstancias en las cuales puede tener lugar la transferencia o cesión de un crédito documentario, en principio posible en relación a cualquier clase de crédito documentario. De acuerdo con el citado precepto, en el supuesto de un crédito transferido, el adquirente o cesionario podrá obtener el pago de su propio crédito contra el envío de sus propios documentos y facturas. Aplicando tal posibilidad al crédito stand-by, ello se traduciría en la posibilidad de que un segundo beneficiario presentara una reclamación afirmando el impago de su propio crédito. La doctrina parece considerar el carácter intuitu personae propio de las garantías independientes a las que resulta asimilable el crédito stand-by, como el factor que haría imposible la transferencia de éste último tipo de crédito.

\section{La identidad de naturaleza entre las garantías independientes y los créditos stand-by y el campo de aplicación de sus respectivas reglamentaciones}

La regulación relativa a los créditos documentarios, que ha conseguido una especial adaptación para servir las necesidades de dicha figura a lo largo de un prolongado período, constituye una referencia

$55 \mathrm{Al}$ respecto, cfr. la exposición de JASINSKY, Credit documentaire, Application aux lettres de credit stand-by des regles et usances, cit., págs. 1116-1120.

${ }_{56} \mathrm{Cfr}$. los promunciamientos de la CCI sobre el citado precepto, Opinions of the ICC Banking Commission 1987-1988, pág. 64, Doc. CCI, 470/Int. 240, 470/531, de 19 de abril de 1988; vid. la exposición de CAPRIOLI, op. cit., pág. 304, nota 85. 
cuando se trata de la fijación del régimen jurídico para la regulación de las garantías independientes, tanto más cuanto que éstas encontraron su inicial desarrollo en forma de créditos stand-by. Dado que su forma es altamente similar y puesto que el carácter de independencia es el principal en ambos casos, la asimilación entre ambas resulta natural, por lo que puede resultar de interés la determinación de aquellos preceptos de las RUU que pudieran ser transpuestas a la regulación de las garantías independientes. Por su parte, la Revisión de 1993 matiza que las RUU resultarán de aplicación a todos los créditos documentarios, incluidos los stand-by, en la medida en que resultaren pertinentes. Sin embargo, no ha tenido lugar una determinación precisa de los preceptos que les pudieran resultar de aplicación, aun cuando determinados artículos han sido añadidos o modificados en atención expresamente a las cartas de crédito stand-by, pese a lo cual la cuestión sigue adoleciendo de imprecisión.

\section{La inaplicabilidad de las RUU a las garantías independientes}

En el supuesto de que una carta de garantía contuviera una referencia a las RUU, los sistemas jurídicos que reconocen el principio de autonomía consideran aquellas como aplicables. Caso de silencio del texto de la garantía, se ha entendido que las RUU no resultan de aplicación, porque se trata de una garantía y no de un crédito documentario ${ }^{57}$. Por su parte, los ordenamientos jurídicos anglosajones o pertenecientes al Common Law podrían sentirse inclinados a la aplicación de las $\mathrm{RUU}^{58}$, dada su afirmación de la identidad de naturaleza entre las diferentes figuras de garantía, aun cuando es claro que un crédito stand-by no documentario (clean) es una garantía no susceptible de regulación por las RUU.

La mayor parte de la doctrina sostiene la inaplicabilidad de las RUU a las garantías independientes. Aquellos autores que mantienen una posición más dubitativa expresan asimismo su impresión de que, incluso en el supuesto de su aplicabilidad, es reducido el número de preceptos aplicables que aportan soluciones, aun cuando éstas son de suyo plenamente evidentes (tal como la afirmación del principio de

57 Cfr. Cabrillac y Mouly, op. cit., pág. 367, y las decisiones jurisprudenciales allí citadas.

58 Cfr. Cabrillac y Mouly, op. cit., nota 147, quienes citan al respecto los casos Wichita Eagle and Beacon Publishing Co. vs. Pacific National Bank, así como el caso Steinmeyer vs. Warner Consolidated Corp; casos asimismo citados por ELLINGER, Letters of Credit en Horn y SCHMitTHOFF, The Transnational law of international commercial transactions, Kluwer, 1982, pág. 241, especialmente pág. 254 (nota 77) y pág. 273. 
independencia), o no resultan de gran utilidad por su ambigüedad (tal como la regulación acerca de la responsabilidad de los bancos) ${ }^{59}$.

De entre los 49 artículos de las RUU, sólo una minoría podrían resultar en principio adecuados para su transposición a la regulación de las garantías independientes.

En un plano general ${ }^{60}$, no resultan de utilidad los preceptos relativos a los documentos, fundamento técnico de los créditos documentarios ausente en las garantías, a los que está dedicada la sección «D». Asimismo, en aquellas otras secciones que inicialmente podrían parecer más ricas en disposiciones con vocación para la regulación de las garantías, tales como la sección «A» que contiene disposiciones generales, la sección «B» relativa a la forma, la sección «E» relativa a la vigencia, pocos son los preceptos adaptados a los problemas y necesidades de las garantías independientes.

Aquellos preceptos que, por no referirse de manera específica al mecanismo de los créditos documentarios, pudiera pensarse son de posible transposición a la regulación de las garantías independientes, no significan precisión o ayuda reales, pues tampoco hacen relación a los problemas principales de las garantías independientes. Tal es el caso de los arts. 12, 16-18, 40-41, 48-49.

En un plano más concreto ${ }^{61}$, algunos preceptos no pueden ser tomados en consideración por su manifiesta inadaptación al mecanismo de las garantías independientes. $1^{\circ}$ ) Ello es lo que sucede con las disposiciones acerca de los bancos confirmadores o notificadores, las cuales carecen de utilidad porque no es habitual, en lo relativo a los créditos stand-by, el recurso a un banco notificador o confirmador. Por otra parte, aun cuando tales fueran las condiciones infrecuentes en que una garantía hubiera podido ser emitida, los arts. 7 y 13 no serían de aplicación porque no reflejan una práctica habitual o establecida en materia de garantías. Incluso en el supuesto de una referencia expresa a las RUU, podría caber duda acerca de la voluntad auténtica de las partes de que tal regla fuera aplicada en razón de lo infrecuente de la situación. $2^{\circ}{ }^{\circ}$ Sería, por otra parte, incoherente la pretensión de aplicación

59 JASINSKY, Credit documentaire... cit., págs. 1116-1120; SiON, «La garantie bancaire internationale et les enseignements du droit americain», en Rev. Banque, 1984, págs. 5 y 20; Cabrillac y Mouly, op. cit., pág. 368, nota 150.

60 Cabrillac y Mouly, op. cit., pág. 369.

61 Cfr. Cabrillac y Mouly, op. cit., págs. 369-372 llevan a cabo un análisis altamente detallado acerca de los preceptos concretos de las RUU que podrían resultar de aplicación a las garantías independientes, para concluir que, o bien los preceptos analizados resultan inaplicables en razón de la teleología de los diferentes preceptos cuya aplicación queda descartada, o bien apreciar la aplicabilidad de determinados preceptos no aporta ventaja alguna. 
del art. 6, el cual permite la declaración de la revocabilidad de un crédito, porque una garantía independiente no puede ser unilateralmente revocada, pues en tal caso perdería todo su valor; por el contrario, las garantías son irrevocables por naturaleza ${ }^{62} .3 .^{\circ}$ ) Otras preceptos, como el art. 14 (a) acerca del derecho de reembolso, o el art. 3 que afirma el principio de independencia de los créditos, contienen soluciones a las que se llegaría igualmente aunque no existieran. $4 .^{\circ}$ ) Algunos preceptos ponen especialmente de manifiesto su inadaptación en razón de las diferencias existentes entre los créditos documentarios y las garantías independientes. Ello es lo que sucede al art. 19 relativo al reembolso entre bancos que resulta inadaptado al mecanismo de la contragarantía; o tal como el art. 6 que permite la emisión de créditos revocables. 5..$\left.^{\circ}\right)$ Por último, determinados preceptos, como los arts. 42 y 47 acerca de la vigencia de los créditos documentarios, ofrecen soluciones que podrían resultar de interés teórico pero resultan inadecuadas a las particularidades de las garantías independientes.

Las consideraciones expuestas permiten concluir que la pretensión de la aplicación de las RUU a las garantías independientes y no documentarias no supone sino una utilidad menor, que quedaría aún más reducida si se toma en consideración el riesgo de que las decisiones e interpretaciones acerca de las garantías pudieran perturbar por analogía el funcionamiento de los créditos documentarios, grandemente asentado después de una larga práctica.

\section{La aplicabilidad de las RUGD a los créditos stand-by}

La CCI se ha pronunciado, aun de manera indirecta, en favor del mantenimiento de la sumisión de los créditos stand-by a las RUU, pues la Introducción de las RUGD indica:

«Standby credits are already governed by the Uniform Customs and Practice for Documentary Credits. They have developed into allpurpose financial support instruments which are used in a much wider range of financial and commercial activity than demand guarantees, and regularly involve practices and procedures that are infrequently encountered in relation to demand guarantees.

Accordingly, while standby credits are technically within the definition of a demand guarantee, it is expected that the issuers of standby credits will continue to use the UCP, which are both more detailed and more appropiate to the particular requirements of standby credits».

62 JASINSKY, art. cit., pág. 1117. 
Sin embargo, opcionalmente y siempre que a ello se hubiera hecho referencia expresa en el texto de la garantía, las RUGD podrán aplicarse a los créditos stand-by pues, de acuerdo con la Introducción de las RUGD, éstas resultan de aplicación:

«to the use of demand guarantees, that is, guarantees, bonds, and other payment undertakings under which the duty of any other documents specified in the guarantee and is not conditional on proof of default by the principal in the underlying transaction».

En relación a la aplicación de las RUGD a los créditos stand-by, la CCI podía haber asumido diversas posibles posiciones: 1.a) la aplicación de las RUU a los créditos stand-by, mientras que las RUGD se aplicarían a otras garantías; $2 .^{a}$ ) el sometimiento de los créditos standby a ambos textos, manteniendo la aplicabilidad de las RUU y sometiéndolos a las nuevas RUGD; $3 .^{\mathrm{a}}$ ) la adopción de un texto específico únicamente relativo a los créditos stand-by, y la exclusión de éstos de la aplicación de las RUU con ocasión de la siguiente revisión de éstas últimas ${ }^{63}$.

Quizá, en busca de una mayor claridad reguladora, hubiera sido preferible elegir la primera solución, al menos en lo relativo a las cartas de crédito stand-by comerciales, puesto que las RUU han cumplido su papel de regulación y de uniformidad en dicha cuestión y, en consecuencia, era deseable que las cartas de crédito stand-by quedaran bajo el campo de aplicación de dichas reglas. No parecía, pues, necesaria la creación de un nuevo texto cuando ya existía uno que había recibido gran aceptación. Sin embargo, es la segunda solución la que ha prevalecido en las RUGD. La Introducción convierte a este texto en aplicable a todas las garantías. El proyecto incluía expresamente las cartas de crédito stand-by que quedarían reguladas a elección de las partes, bien por las RUU, bien por las RUGD. El interés de la posibilidad de sumisión a ambos textos encuentra su fundamento en la distinción entre las cartas de crédito stand-by comerciales, reguladas por las RUU, y las cartas de crédito stand-by financieras, reguladas por las RUGD.

En cualquier caso, tal como quedó apuntado anteriormente, si las ISP 98 terminaran por alcanzar implantación generalizada, las cuestiones derivadas de la actual coexistencia de diferentes reglamentaciones, cuyos respectivos campos de aplicación se solapan entre sí, quedarían clarificados en buena medida.

63 Cfr. CAPRIOLI, Le credit documentaire... cit., pág. 316. 